\title{
К ВОПРОСУ РАЗВИТИЯ АЛЬТЕРНАТИВНОЙ ЭНЕРГЕТИКИ В РОССИЙСКОЙ ФЕДЕРАЦИИ
}

\author{
(c) 2021 Салихов Ирек Фаритович \\ кандидат экономических наук, доцент, председатель Совета директоров АО «ЯДРАН» \\ Высшая школа бизнеса \\ Казанский (Приволжский) федеральный университет, Россия, Татарстан, Казань \\ E-mail: mbakazan@kpfu.ru
}

В статье автора рассматривается вопрос развития альтернативной энергетики в Российской Федерации. Объектом исследования выступает альтернативная энергетика в Российской Федерации, а предметом - ее развитие во всех возможных аспектах. Теоретическое значение исследования заключено в углубленном изучении вопроса развития сложных многокомпонентных (в том числе энергетических) систем на федеральном уровне. Также теоретическое значение исследования определяется усовершенствованием методической базы анализа сложных процессов в динамике. Практическое и прикладное значение исследования заключены в определении перспективных направлений развития альтернативной энергетики в Российской Федерации на ближайшие пять лет с указанием количественно определенных приоритетов. Научные и практические результаты, полученные в процессе раскрытия анализируемого вопроса могут быть использованы для решения широкого круга задач в области экономики и энергетики.

Ключевые слова: альтернативная энергетика, вопросы, развитие, динамика, эффективность, направления, возобновляемый источник энергии, Российская Федерация.

С развитием мирового финансового кризиса, усиленного последствиями глобальной пандемии, вызванной беспрецедентным распространением, коронавируса [3, с. 159], достаточно значимую актуальность приобретают вопросы устойчивого развития $[1$, с. 5] всех без исключения глобальных систем, включая социальную, экономическую и энергетическую.

По мнению крупнейших мировых консалтинговых компаний $[7,8]$ процесс устойчивого развития [1,2] мировой энергетической системы невозможен без развития альтернативной энергетики, призванной, в первую очередь, снизить углеродный след человечества как минимум до приемлемых для планеты Земля значений, а в идеальном случае сделать его нулевым.

Именно поэтому во многих странах мира, включая Российскую Федерацию, вопросы развития альтернативной энергетики [4, с. 207] выходят на один из первых планов глобальных стратегических карт.

Рассматривая вопрос альтернативной энергетики применительно к Российской Федерации, можно отметить, что ее значимость на мировой арене в вопросах генерации энергии из возобновляемых источников достаточно незначительная [5, с. 221]. Так, согласно данным исследований, проведенных компанией British Petroleum [6], объемы производства энергии из возобновляемых источников (в тераватт - часах) для Российской Федерации, Европы и в глобальном масштабе за 2020 год приведены на рисунке 1.

На основе данных рисунка 1 можно заключить, что доля генерации из возобновляемых источников в Российской Федерации в 2020 году, относительно Европы, составила порядка 0,38 процента (3,5 ТВт-ч против 921,0 ТВт-ч), а относительного всего Мира лишь 0,11 процента (3,5 ТВт-ч против 3147,0 ТВт-ч). Доля генерации возобновляемой энергии в Европе относительно всего Мира составила при этом порядка 29,26 процента.

Целевое рассмотрение динамического аспекта вопроса локально для Российской Федерации за последние 10 лет (рисунок 2) позволяет сделать вывод, что объем производства энергии из возобновляемых источников увеличился более чем в 5,8 раза (на 2,9 ТВт-ч). Сам процесс развития исследуемого показателя в 20112020 годах протекал преимущественно поступательно (с 2012 по 2013 годы имел место нулевой результат изменения, а с 2019 по 2020 годы, практически двукратный прирост) и характеризовался в основном положительной динами- 


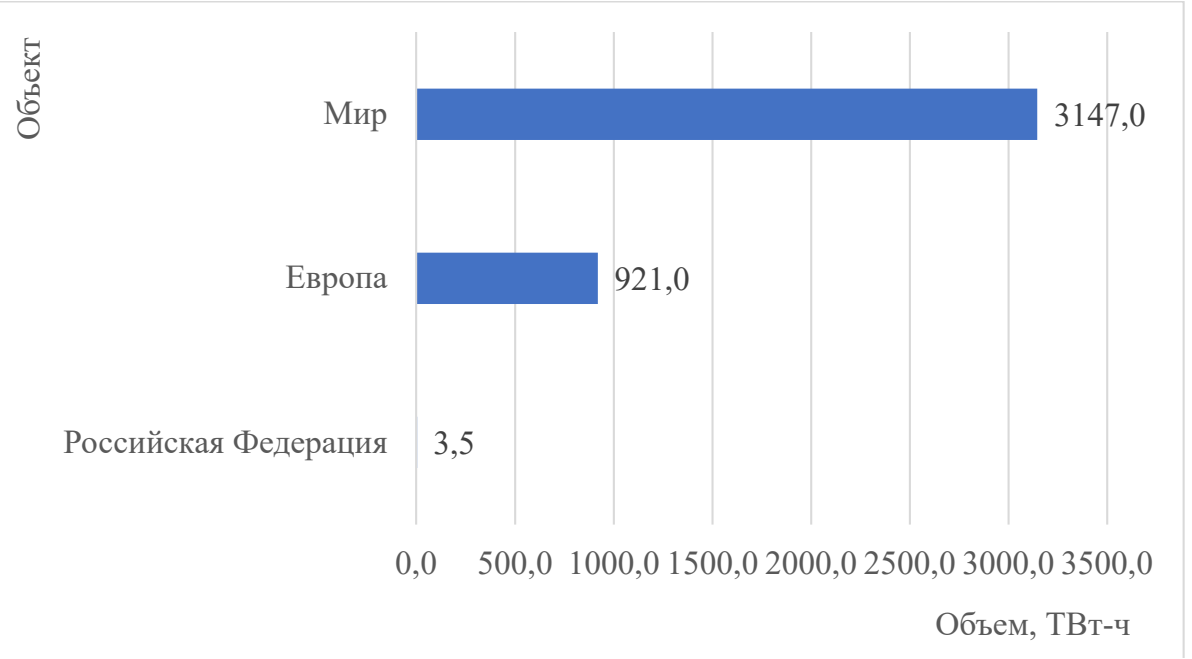

Puc. 1. Данные по объемам производства энергии из возобновляемых источников (в тераватт - часах) для Российской Федерации, Европы и в глобальном масштабе в 2020 году [6]

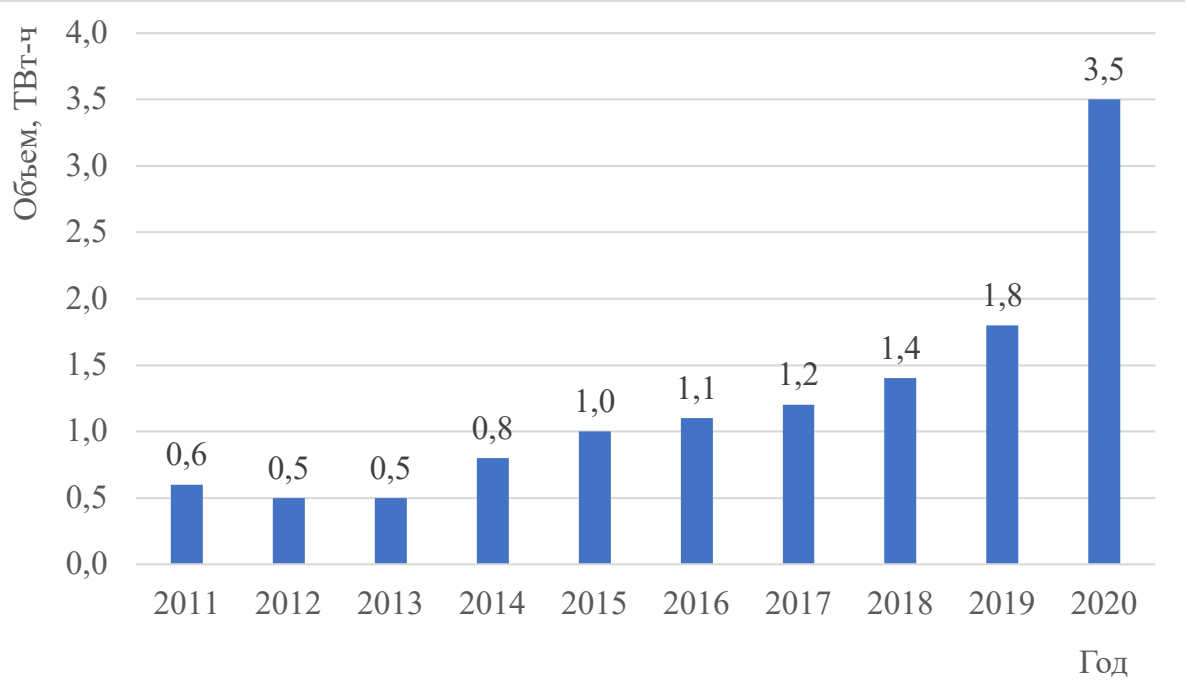

Puc. 2. Данные по объемам производства энергии из возобновляемых источников (в тераватт - часах) для Российской Федерации в 2011-2020 годах [6]

кой (за исключением периода с 2011 по 2012 год, в котором имело место снижением объема на 0,1 единицы).

Основываясь на приведенных данных, можно сделать вывод, что несмотря на незначительные масштабы относительно глобального пространства, развитие альтернативной энергетики в Российской Федерации происходит достаточно активно, что стало особенно заметно в 2020 году.

При этом по предварительным данным, по итогам 2021 года объем производства энергии из возобновляемых источников может приблизиться к отметке в 4,5 ТВт-ч. Однако, несмотря на столь значимые величины, доля указанных источников в совокупном объеме генерации в Российской Федерации все еще не превышает одного процента (в конце 2021 года значение данного показателя может составить лишь 0,9 процента).

По мнению автора, для достижения более значимых в мировом масштабе результатов в рамках альтернативной энергетики и реализации программы устойчивого развития, объем энергии, получаемой из возобновляемых источников в Российской Федерации в ближайшие пять лет необходимо значительно увеличить. Ввиду этого, до 2025 года предлагается рассмотреть три сценария развития альтернативной энергетики, характеризующихся различными уровнями производства энергии из возобнов- 
ляемых источников с начальным горизонтом в 2021 году (таблица 1).

Целевые объемы производства энергии из возобновляемых источников (в тераватт - часах) для Российской Федерации на 2021-2025 годы в рамках пессимистичного сценария приведены на рисунке 3 .

Из рисунка 3 можно увидеть, что пессимистичный сценарий развития опирается на финальное значение объема производства энергии из возобновляемых источников к 2025 году в размере 10 ТВт-ч. Данный показатель для Российской Федерации, исходя из текущей ситуации и объемов ввода в эксплуатацию установок, в рамках программ развития солнечной энергии является вполне достижимым. Вместе с тем реализация сценария в пессимистичной версии не позволит в полной мере значимо изменить положение Российской Федерации на мировой арене. Совокупная вероятность реализации данного сценария развития оценена авторов в 34,9 процента.

Целевые объемы производства энергии из возобновляемых источников (в тераватт - часах) для Российской Федерации на 2021-2025 годы в рамках нейтрального сценария приведены на рисунке 4.

Характеризуя нейтральный сценарий развития, отметим, что, учитывая наивысший уровень его вероятности реализации среди рассматриваемых (39,8 процента против 34,9 процента для пессимистичного), Российская Федерация может достичь целевых значений обозначенных в нем показателей по выработке.

Целевые объемы производства энергии из возобновляемых источников (в тераватт - ча-

Таблица 1. Разработанные сценарии развития альтернативной энергетики для Российской Федерации на 2021-2025 годы

\begin{tabular}{|c|l|c|}
\hline $\begin{array}{c}\text { Наименование } \\
\text { сценария }\end{array}$ & \multicolumn{1}{|}{$\begin{array}{c}\text { Характеристика } \\
\text { сценария }\end{array}$} & $\begin{array}{c}\text { Оцененная } \\
\text { вероятность } \\
\text { реализации,\% }\end{array}$ \\
\hline Пессимистичный & $\begin{array}{l}\text { Характеризует объемы производства энергии из альтернативных } \\
\text { источников при наихудшем стечение обстоятельств в будущем }\end{array}$ & 34,9 \\
\hline Нейтральный & $\begin{array}{l}\text { Характеризует объемы производства энергии из альтернативных } \\
\text { источников при нейтральном стечение обстоятельств в будущем }\end{array}$ & 39,8 \\
\hline Оптимистичный & $\begin{array}{l}\text { Характеризует объемы производства энергии из альтернативных } \\
\text { источников при нейтральном стечение обстоятельств в будущем }\end{array}$ & 25,3 \\
\hline Совокупная вероятность реализации,\% & 100,0 \\
\hline
\end{tabular}

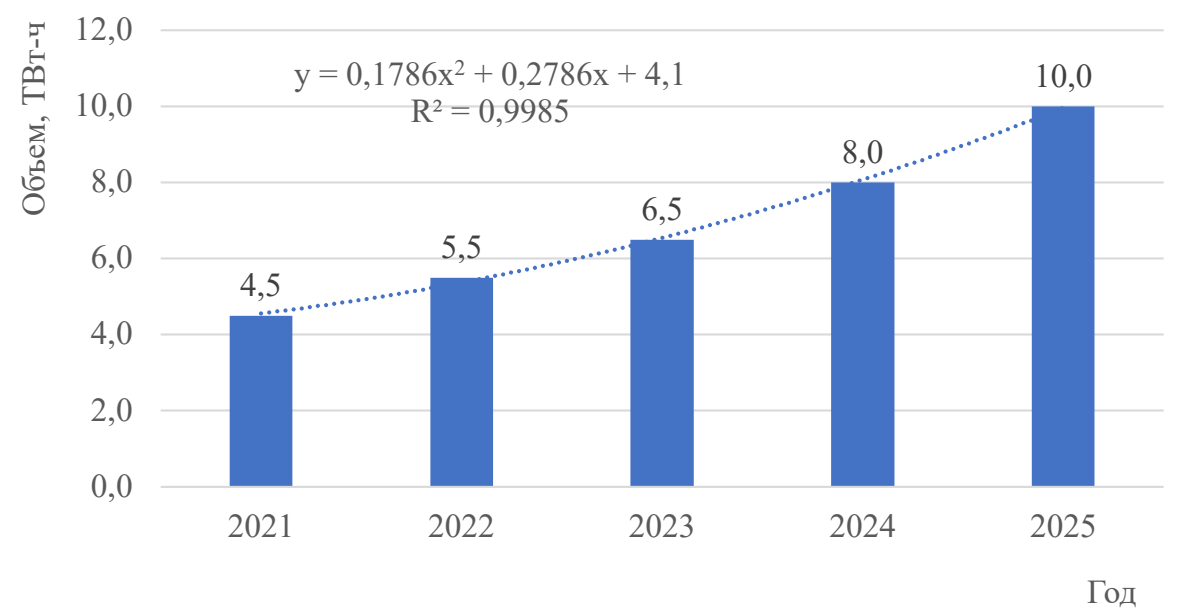

Puc. 3. Целевые объемы производства энергии из возобновляемых источников (в тераватт - часах) для Российской Федерации на 2021-2025 годы (пессимистичный сценарий развития) Источник: составлено автором самостоятельно 


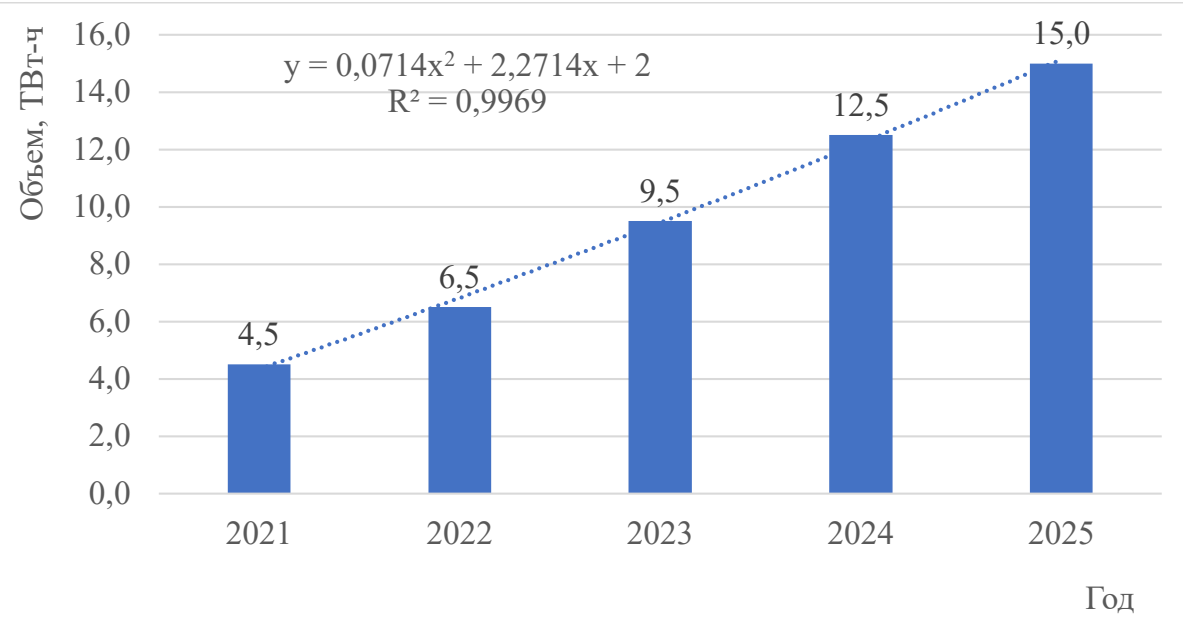

Рuc. 4. Целевые объемы производства энергии из возобновляемых источников (в тераватт - часах) для Российской Федерации на 2021-2025 годы (нейтральный сценарий развития) Источник: составлено автором самостоятельно

сах) для Российской Федерации на 2021-2025 годы в рамках оптимистичного сценария приведены на рисунке 5.

По оптимистичному сценарию можно отметить, что, если Российская Федерация достигнет объема производства энергии, получаемой из возобновляемых источников в 20,0 МВт-ч к 2025 году, то ее показатели на мировой арене улучшатся относительно достигнутых уже более значимо. Однако, учитывая уровень совокупной вероятности реализации лишь в 25,3 процента из 100 процентов данный сценарий в полной мере реализован не будет.

Таким образом, можно отметить, что устойчивое развитие Российской Федерации в целом и ее энергетической системы в частности невозможно без активизации использования энергии из возобновляемых источников, сбалансиро- ванной как с текущей экономической ситуацией, так и с технологическими возможностями страны в целом.

Учитывая положительную десятилетнюю динамику производства энергии из возобновляемых источников, можно сделать вывод, что Российская Федерация сделала огромный скачек, уже активизировав такие направления, как энергия солнца и ветра. Однако, учитывая относительную низкую долю произведенной зеленой энергии в федеральном формате, до сих пор стремящуюся к отметке лишь в один процент, имеет смысл задуматься о реализации ряда инициатив, направленных на разрешение сложившейся ситуации и о значительном увеличении объемов использования возобновляемых энергетических ресурсов.

Такими инициативами, нацеленными, в пер-

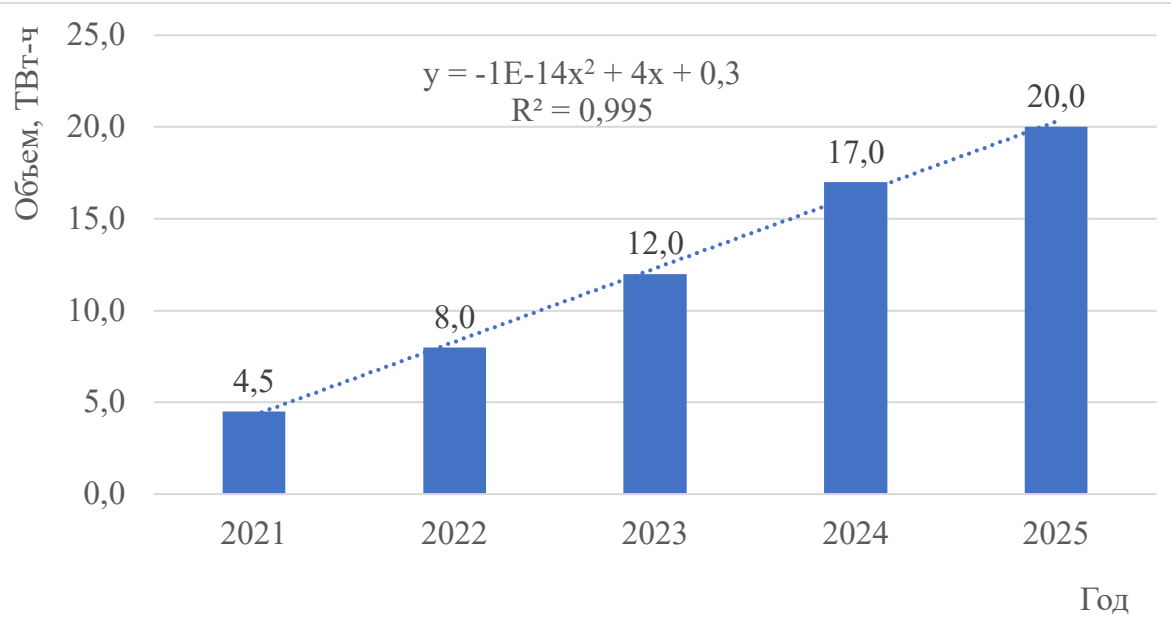

Puc. 5. Целевые объемы производства энергии из возобновляемых источников (в тераватт - часах) для Российской Федерации на 2021-2025 годы (оптимистичный сценарий развития) Источник: составлено автором самостоятельно 
вую очередь, на достижение целевых значений объемов производства энергии из возобновляемых источников могут стать:

- введение новых дополнительных производственных мощностей в рамках проектов по солнечной энергетике в целевых регионах Российской Федерации с высоким количеством эффективных солнечных дней;

- реализация комплекса мер, нацеленных на снижение стоимости оборудования, используемого для производства солнечной энергии различными категориями потребителей (например, за счет заключения стратегических соглашений в данной сфере с передовыми производителями в зарубежных странах);

- создание на федеральном уровне простой, доступной и одновременно универсальной методики внедрения инновационного оборудования, используемого для производства солнечной энергии различными категориями потребителей (например, за счет изучения зарубежного опыта в данном вопросе Китая и Японии).

\section{Библиографический список}

1. Ахметшина А.Р. Оценка уровня функционирования институциональной среды устойчивого развития в России / А.Р. Ахметшина / Управление устойчивым развитием - Казань: Изд-во: ФГБОУ ВПО «КНИТУ», 2021. № 4 (35). C. 5-10.

2. Ахметшина А. Р., Сотов Д.И. Реализация принципов устойчивого развития в энергетической отрасли Республики Татарстан / А. Р. Ахметшина, Д. И. Сотов / Проблемы современной экономики - Санкт-Петербург: Изд-во ООО НПК «РОСТ». 2018. № 4 (68). С. 158-161.

3. Иванова Д.Г. Устойчивое развитие мировой экономики в условиях пандемии / Д.Г. Иванова / Экономические науки - Москва: Изд-во: ООО «24 Принт», 2021. - № 6. - С. 159-162.

4. Сосюра Б.Е. Тенденции и перспективы развития нетрадиционной электроэнергетики / Б.Е. Сосюра / Вопросы устойчивого развития общества - Махачкала: Изд-во: ООО «Институт развития образования и консалтинга», 2021. - № 1. - С. 207-213.

5. Тимчук B. В. Альтернативные источники энергии. Анализ альтернативной энергетики в России / В.В. Тимчук / Аспирант - Ростов-на-Дону: Изд-во: ООО «Приоритет», 2021. - № 1. - С. 221-223.

6. Компания «British Petroleum plс» [Электронный ресурс]: Statistical Review of World Energy 2021 - Официальный сайт компании «British Petroleum plc», 2021. - Режим доступа: https://www.bp.com/

7. Компания «Ernst\&Young» [Электронный ресурс]: обзоры информации для клиентов - Официальный сайт компании «Ernst\&Young», 2021. - Режим доступа: https://www.ey.com/ru_ru

8. Компания «PWC» [Электронный ресурс]: материалы и обзоры - Официальный сайт компании «РWC», 2021. - Режим доступа: https://www.pwc.ru/ 